\title{
Drogas entre adolescentes e adultos jovens: estudo com professores de um assentamento do Brasil Central
}

\begin{abstract}
RESUMO | Objetivo: identificar entre os professores de um assentamento do Brasil Central a discussão sobre o tema drogas entre adolescentes e adultos jovens. Método: estudo de corte transversal realizado no período de fevereiro de 2017 a maio de 2018 realizado com 21 professores com mais de seis meses de atuação na escola, de uma Instituição Pública de Ensino de um assentamento da reforma agrária. Resultados: Sobre os malefícios do uso de drogas, a maioria $(76,2 \%)$ responderam que conhecem. Quanto à aquisição de informação sobre alunos usuários fazendo uso de drogas dentro, ou nas proximidades, do ambiente escolar pelos docentes foi relatado por mais da metade dos investigados. Conclusão: Acredita-se que os gestores, impulsionados pelos achados da pesquisa, em parceria com as instituições de ensino e saúde do assentamento, consigam implementar e desenvolver discussões sobre uso e malefícios das drogas entre os professores, estudantes e familiares residentes no assentamento.
\end{abstract}

Palavras-chaves: Drogas; Professor; Adolescente.

\begin{abstract}
Objective: to identify among the teachers of a settlement in Central Brazil the discussion on the topic of drugs among adolescents and young adults. Method: cross-sectional study carried out from February 2017 to May 2018 conducted with 21 teachers with more than six months of experience in school, from a Public Teaching Institution in an agrarian reform settlement. Results: Regarding the harm of drug use, the majority (76.2\%) answered that they know. As for the acquisition of information about student users using drugs inside or near the school environment by teachers, it was reported by more than half of those investigated. Conclusion: It is believed that managers, driven by the research findings, in partnership with the educational and health institutions of the settlement, will be able to implement and develop discussions about the use and harm of drugs among teachers, students and family members residing in the settlement.
\end{abstract}

Keywords: Drugs; Teacher; Teenager.

RESUMEN | Objetivo: identificar entre los docentes de un asentamiento en el centro de Brasil la discusión sobre el tema de las drogas entre adolescentes y adultos jóvenes. Método: estudio transversal realizado entre febrero de 2017 y mayo de 2018 realizado con 21 docentes con más de seis meses de experiencia en la escuela, de una Institución de Enseñanza Pública en un acuerdo de reforma agraria. Resultados: Respecto al daño del uso de drogas, la mayoría (76.2\%) respondió que lo saben. En cuanto a la adquisición de información sobre los usuarios estudiantes que usan drogas dentro o cerca del entorno escolar por parte de los maestros, más de la mitad de los investigados informaron de ello. Conclusión: Se cree que los gerentes, impulsados por los resultados de la investigación, en asociación con las instituciones educativas y de salud del asentamiento, podrán implementar y desarrollar discusiones sobre el uso y el daño de las drogas entre los maestros, estudiantes y familiares que residen en el asentamiento.

Palavras claves: Drogas; Profesor; Adolescente.

Lucíola Silva Sandim

Enfermeira. Mestre em Cuidado em Enfermagem. Docente da Universidade Estadual de Goiás. Câmpus Itumbiara e UNICERRADO Centro Universitário de Goiatuba. https://orcid. org/0000-0002-6541-0014

\section{Stéfany Martins Silva}

Enfermeira. Mestre em Assistência e Avaliação em Saúde pela Faculdade de Enfermagem da Universidade Federal de Goiás. Docente pela Secretaria de Educação do Distrito Federal. https://orcid.org/00000002-9533-4833

\section{Breno Marçal de Araújo}

Acadêmico de Enfermagem. Universidade Estadual de Goiás. Câmpus Itumbiara. https://orcid.org/0000-0002-9774-8618

\section{Paulie Marcelly Ribeiro dos Santos}

Enfermeira. Doutoranda em Cuidado em Enfermagem. Programa de Pós-graduação em Enfermagem. Faculdade de Enfermagem da Universidade Federal de Goiás. https://orcid. org/0000-0003-0056-364X

\section{Elisângela Franciscon Naves}

Enfermeira. Mestre em Cuidado em Enfermagem. Docente da Universidade Estadual de Goiás. Câmpus Itumbiara. https://orcid.org/0000-0002-0847-0440

\section{Marcos André de Matos}

Enfermeiro. Doutor em Ciências da Saúde. Docente da Faculdade de Enfermagem da Universidade Federal de Goiás. https:// orcid.org/0000-0001-8643-7032
INTRODUÇÃO

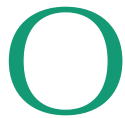
consumo de drogas lícitas e ilícitas entre a população jovem, desde a década de 1960, representa um grande problema multifacetado de saúde pública mundial, exigindo avaliações frequentes. Estima-se que 29 milhões da população mundial fazem uso de drogas em todo o mundo $(1,2)$. Dentre a população jovem, os assentados podem ser especialmente vulneráveis ao uso e consequências negativas dessas substâncias devido a um maior descaso sociocultural, infraestrutura de habitação, emprego precário e acesso limitado a programas de intervenção ${ }^{(3-5)}$, em especial pelos equipamentos sociais, como as unidades de saúde e de educação. Os assentamentos, conjunto de unidades agrícolas, foram idealizados, 
principalmente, para abrigar famílias carentes advindas de áreas irregulares e de preservação ambiental.

Estudo conduzido nos Estados Unidos da América $^{(3)}$ evidenciou que 30\% dos jovens assentados tinham história de consumo excessivo de bebidas alcoólicas. No Piauí, estimou em 11,67\% a prevalência de uso abusivo de álcool, utilizando a escala Alcohol Use Disorders Identification Test (AUDIT) ${ }^{(6)}$. Já investigação em assentamento da região Centro-oeste do Brasil (7) estimou em 27,6\% (IC95\% 20,0-36,9\%) o uso de drogas ilícitas, dentre elas maconha, cocaína, crack, LSD e inalantes foi relatado por $24,8 \%(95 \mathrm{Cl} 17,5-33,8 \%)$, $6,7 \% \quad(95 \mathrm{Cl} \quad 3,3-13,1 \%), 2,9 \% \quad(95 \mathrm{Cl}$ $1,0-8,1 \%), \quad 1,9 \% \quad(95 \mathrm{Cl} \quad 0,5-6,7 \%)$ e $1,0 \%(95 \mathrm{Cl} \quad 0,2-5,2 \%)$ dos participantes, respectivamente.

Globalmente, já existem fortes evidências de que as instituições de ensino sempre foram, e são consideradas imprescindíveis agentes na prevenção do uso de drogas entre os adolescentes e adultos jovens, pois, os professores são potenciais veículos, tanto de formação, quanto de informação passível de mudança de comportamentos e atitudes de risco ${ }^{(8,9)}$. Todavia, há décadas, os programas institucionais não têm conseguido ter êxito, muito provavelmente devido à inabilidade dos professores em abordar tal temática repleta de estigma em um ambiente, em geral, ainda, tradicional e repressor, como também pela precariedade do sistema educacional, falta de projetos preventivos, processos familiares fragilizados e participação ativa dos pais e/ou responsáveis pelos jovens ${ }^{(10-12)}$.

Desde 2006, o Brasil legitima a necessidade de formação dos professores e a implantação de projetos pedagógicos voltados para a prevenção de drogas ${ }^{(11)}$. Nos dias atuais, o Ministério da Saúde e o da Educação vem incentivando a discussão do tema por meio do Programa Saúde na Escola (PSE) ${ }^{(12)}$. Nesse contexto, inúmeros foram os estudos concernentes à identificação e avaliação dessas políticas, como já descrito pelo Sistema Nacional de Políticas Públicas sobre Drogas, onde há baixa adesão do PSE ${ }^{(10)}$, mas, até o momento, nenhuma com professores

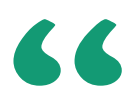

Globalmente, já existem fortes evidências de que as instituições de ensino sempre foram, e são consideradas imprescindíveis agentes na prevenção do uso de drogas entre os adolescentes e adultos jovens, pois, os professores são potenciais veículos, tanto de formação, quanto de informação passível de mudança de comportamentos $\mathrm{e}$ atitudes de risco. de instituições de ensino localizadas em assentamento. Assim, a presente investigação objetivou identificar entre os professores de um assentamento do Brasil Central a discussão sobre o tema drogas entre adolescentes e adultos jovens

MÉTODO

Trata-se de um estudo de corte transversal realizado no período de fevereiro de 2017 a maio de 2018, utilizando os preceitos da epidemiologia social. Participaram da pesquisa 21 professores de uma Instituição Pública de Ensino de um assentamento da reforma agrária localizada na Região Central do Brasil. Foram elegíveis os que possuíam mais de seis meses de atuação na escola e excluídos os em licença médica e férias.

Todos os elegíveis foram convidados a participar do estudo e orientados sobre a natureza, objetivos, metodologia, riscos e benefícios, e após foi oferecido o Termo de Consentimento Livre e Esclarecido (TCLE) para leitura e assinatura.

A coleta de dados ocorreu em local privativo nas dependências da instituição de ensino, utilizando-se um roteiro estruturado, composto por quatro grupos de questões: 1) características sócio demográficas e laborais; 2) Consumo de álcool e uso de drogas na adolescência 3) Discussão sobre drogas na escola e em sala de aula 4) Vulnerabilidade dos assentados às drogas. Todas as variáveis descritas acima foram apresentadas por meio de tabelas. Ainda, visando o sigilo e maior confiabilidade das informações obtidas, todos os auxiliares de pesquisa receberam treinamento.

Os dados obtidos, após validação e digitação em microcomputador, foram analisados pelo programa estatístico Statistical Package for the Social Sciences, versão 17.0. Para as variáveis contínuas foram calculadas médias e desvio-padrão. Este estudo foi precedido da aprovação pelo Comitê de Ética e Pesquisa da Universidade Federal de Goiás, sob registro no365/11. 
Tabela 1- Características sociodemográficas de professores de adolescentes escolares de uma área de assentamento do Brasil Central, 2017-2018

\begin{tabular}{lcc} 
Características & N & $\%$ \\
Sexo & & \\
\hline Masculino & 6 & 28,57 \\
\hline Feminino & 15 & 71,43 \\
\hline
\end{tabular}

Idade média 31,2 anos $(\mathrm{DP} \pm 4,32)$

\begin{tabular}{|c|c|c|}
\hline $24-33$ anos & 8 & 38,10 \\
\hline $34-43$ anos & 8 & 38,10 \\
\hline $44-52$ anos & 5 & 23,80 \\
\hline \multicolumn{3}{|l|}{ Estado civil } \\
\hline Solteiro & 7 & 33,34 \\
\hline Casado & 11 & 52,38 \\
\hline Divorciado & 3 & 14,28 \\
\hline \multicolumn{3}{|l|}{ Religião } \\
\hline Católico & 8 & 38,10 \\
\hline Evangélico & 6 & 28,57 \\
\hline Espírita & 5 & 23,80 \\
\hline Outros & 2 & 9,53 \\
\hline \multicolumn{3}{|c|}{ Renda familiar média 6,2 SM $(\mathrm{DP} \pm 1,02)$} \\
\hline 2 - 4 SM & 5 & 23,81 \\
\hline 5- 7 SM & 6 & 28,57 \\
\hline $8-10$ SM & 5 & 23,81 \\
\hline$>10 \mathrm{SM}$ & 5 & 23,81 \\
\hline
\end{tabular}

SM: Salário Mínimo

Tabela 2- Características pedagógicas de professores de adolescentes escolares de uma área de assentamento do Brasil Central, 2017-2018

\begin{tabular}{lcc|} 
Características & N & $\%$ \\
\hline Área de formação & & \\
\hline Pedagogia & 11 & 52,38 \\
\hline Letras & 2 & 9,53 \\
\hline Matemática & 1 & 4,76 \\
\hline Biologia & 2 & 9,53 \\
\hline Educação física & 3 & 14,28 \\
\hline Artes & 1 & 4,76 \\
\hline História & 1 & 4,76 \\
\hline Tempo de formação média 9,7 anos (DP \pm 3,13) & & \\
\hline 2-8 anos & 12 & 57,14 \\
\hline 9-15 anos & 7 & 33,33 \\
\hline$>16$ Anos & 2 & 9,53 \\
\hline Pós-graduação & & \\
\hline
\end{tabular}

\section{RESULTADOS}

A Tabela 1 apresenta as características sócio demográficas investigados, sendo a maioria do sexo feminino $(71,43 \%)$, casada $(52,38 \%)$ e se denominavam católicos $(38,10 \%)$. Grande parcela dos entrevistados possuía entre 24 a 43 anos de idade (76,18\%). Quanto à renda familiar a maioria $(52,38 \%)$ recebia entre 2 a 7 salários mínimos.

A Tabela 2 mostra as características pedagógicas dos professores. Sobressaíram aqueles formados em pedagogia $(52,38 \%)$ e com até oito anos de formação $(57,14 \%)$, sendo que $(80,95 \%)$ declararam possuir pós-graduação. Concernente à carga horária de trabalho, $12(44,15 \%)$ professores ministravam mais de $40 \mathrm{~h} / \mathrm{semanais}$ de aulas.

A Tabela 3 demonstra a percepção acerca da temática álcool e outras drogas. Apesar de todos acreditarem na importância da orientação dos estudantes quanto temática, somente $71,43 \%$ desses já abordaram ou abordam em suas aulas. Para muitos $(61,9 \%)$ a idade ideal para se discutir o tema entre os 10 e 13 anos e (76,2\%) percebem que os estudantes possuem conhecimento sobre os malefícios do uso de drogas. Dos entrevistados $(52,38 \%)$ relataram ter conhecimento de aluno ser usuário de drogas, $(61,9 \%)$ percebem que os alunos apresentam comportamento de risco e 19,05\% já vivenciaram alunos se drogando nas dependências da escola ou entorno.

A Figura 1 apresenta a percepção dos investigados acerca da responsabilidade em abordar a temática no assentamento. A maioria $(90,48 \%)$ acredita que cabe mãe a responsabilidade de orientação e $85,72 \%$ creditam a responsabilidade em discutir álcool e drogas com os adolescentes e jovens aos pais. Somente $14,28 \%$ dos entrevistados afirmaram ser de responsabilidade da escola.

\section{DISCUSSÃo}

Os professores do assentamento apresentaram algumas características discrepantes ao comparar o Censo Escolar do 


\begin{tabular}{|lcc|}
\hline Sim & 17 & 80,95 \\
\hline Não & 4 & 19,05 \\
\hline Disciplina que ministra & & \\
\hline Várias & 10 & 47,62 \\
\hline Inglês & 1 & 4,76 \\
\hline Matemática & 1 & 4,76 \\
\hline Biologia & 2 & 9,53 \\
\hline Geografia & 1 & 4,76 \\
\hline Libras & 1 & 4,76 \\
\hline Educação fisica & 3 & 14,29 \\
\hline Português & 1 & 4,76 \\
\hline História & 1 & 4,76 \\
\hline Tempo de trabalho na Escola do assentamento & & \\
\hline$<1$ ano & 8 & 38,10 \\
\hline $1-2$ anos & 7 & 33,33 \\
\hline $3-4$ anos & 6 & 28,57 \\
\hline Carga horária de trabalho semanal & & \\
\hline$<40 \mathrm{~h}$ & 9 & 42,85 \\
\hline $40-60 \mathrm{~h}$ & 9 & 42,85 \\
\hline $70-90 \mathrm{~h}$ & 3 & 14,30 \\
\hline
\end{tabular}

$\mathrm{H}$ : horas

Tabela 3: Percepção acerca da temática álcool e outras drogas segundo professores de adolescentes escolares de uma área de assentamento do Brasil Central, 2017-2018.

\begin{tabular}{|c|c|c|}
\hline Questões & $\mathrm{N}$ & $\%$ \\
\hline \multicolumn{3}{|c|}{$\begin{array}{l}\text { Considera importante orientação quanto às drogas e álcool na } \\
\text { escola }\end{array}$} \\
\hline Sim & 21 & 100 \\
\hline Não & - & - \\
\hline
\end{tabular}

Idade ideal para abordar a temáticas drogas e álcool na escola

\begin{tabular}{lccc|}
\hline 7 - 9 anos & 8 & 38,1 \\
\hline $10-13$ anos & 13 & 61,9 \\
\hline Já abordou ou aborda os temas drogas e álcool em sala de aula & & \\
\hline Sim & 15 & 71,43 \\
\hline Não & 6 & 28,57 \\
\hline
\end{tabular}

Percebe conhecimento dos alunos quanto aos malefícios de uso de drogas

\begin{tabular}{|lcc|}
\hline Sim & 16 & 76,2 \\
\hline Não & 5 & 23,8 \\
\hline Tem conhecimento de algum aluno que seja usuário de drogas & & \\
\hline Sim & 11 & 52,38 \\
\hline Não & 9 & 47,62 \\
\hline
\end{tabular}

Ministério da Educação ${ }^{(21)}$, pois verificou-se tratar de um grupo mais jovem, com menor tempo de formação e com maior proporção de homens, podendo inferir que os professores em início de carreira procuram locais com maior carência de profissionais até adquirirem experiência. Quase a totalidade $(80,95 \%)$ referiu pós-graduação, taxa muito superior à de $34 \%$ encontrada em Goiás ${ }^{(13)}$. Acredita-se que esse achado reflita nos indicadores de educação da Região, na qual os profissionais estão buscando qualificação cada vez mais precoce em busca de melhor remuneração ${ }^{14}$.

Assim, acredita-se que estes profissionais, se estimulados pelos gestores, colegas de trabalho e rede social do adolescente, possuem competência para incitar a discussão sobre o uso de drogas, tanto na escola, quanto no assentamento, minimizando, sobremaneira, as consequências do uso de drogas, como evasão escolar, reprovações, violência, processos familiares interrompidos, estresse, outros.

Quanto à renda familiar a maioria, recebia menos de sete salários mínimos e $47,62 \%$ ministravam várias disciplinas. Estudos indicam que a remuneração está associada com a qualidade de vida do profissional, o que pode influenciar no vínculo com os estudantes em sala de aula e, consequentemente na abertura para o diálogo sobre temas conflitantes vivenciados pelos jovens ${ }^{(15-17)}$. Tais achados merecem atenção dos gestores, uma vez que prejudicam, sobremaneira, a identificação de déficits educacionais dos alunos, as dificuldades pedagógicas da escola e os problemas sociais da comunidade, reduzindo a capacidade do professor em elaborar e executar projetos de prevenção de drogas, propondo soluções e, mesmo atuando na resolução da situação vivenciada no ambiente intra e extraescolar ${ }^{(18,10)}$.

Todos os investigados acreditam na importância de orientar os estudantes quanto ao uso de álcool e outras drogas na escola, todavia, somente $71,43 \%$ já o fizeram, e de forma superficial em sala 


\section{Alunos apresentam comportamento de risco para o uso de drogas e álcool}

\begin{tabular}{|lcc|}
\hline Sim & 13 & 61,90 \\
\hline Não & 7 & 33,34 \\
\hline Não sabe & 1 & 4,76 \\
\hline Acha necessário abordar a temática de drogas na sala de aula & & \\
\hline Sim & 19 & 90,48 \\
\hline Não & 1 & 4,76 \\
\hline Não sabe & 1 & 4,76 \\
\hline Vivenciou alunos se drogando na escola & & \\
\hline Sim & 4 & 19,05 \\
\hline Não & 16 & 76,19 \\
\hline Não sabe & 1 & 4,76 \\
\hline
\end{tabular}

Fonte: Própria.

Figura 1: Responsabilidade pela orientação sobre uso de álcool e outras drogas segundo professores de adolescentes escolares de uma área de assentamento do Brasil Central, 2017-2018

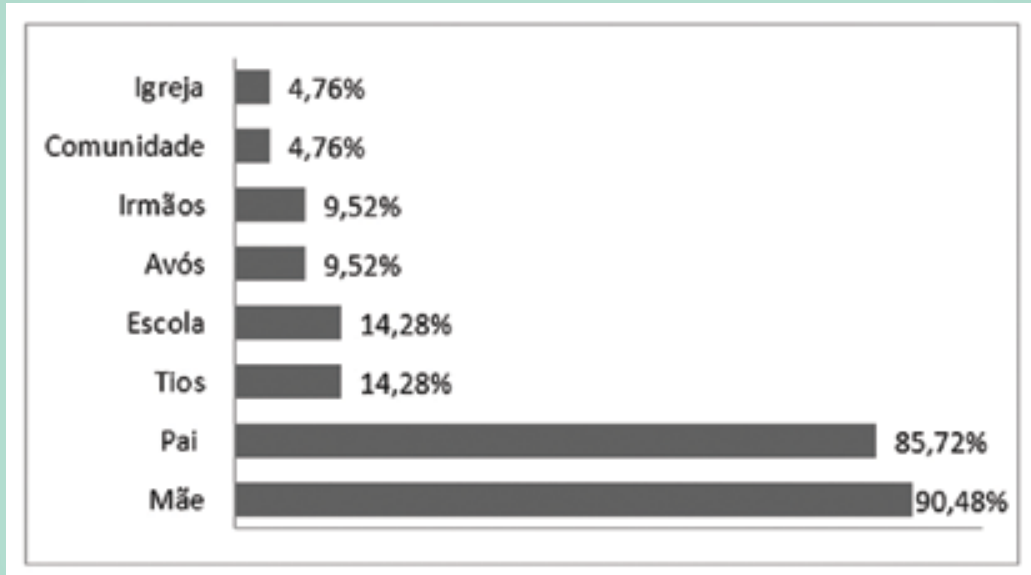

de aula. Abordar drogas e álcool somente em sala de aula é diferente em abordar na escola, uma vez que na sala de aula o professor assume a responsabilidade sozinho, sem o apoio da instituição ou de um programa de apoio que torne a estratégia institucional e plausível de execução ${ }^{(19)}$.

Dos investigados, 90,48\% e 85,72\% referiram que a educação sobre o tema é de responsabilidade materna e paterna, respectivamente. Esse achado deve ser explorado minuciosamente em estudos com abordagem qualitativa, pois, muito possivelmente remete uma visão preconcebida de que te-
É extremamente necessário o investimento na qualificação dos professores sobre drogas e álcool, permitindo que contribuam de forma efetiva e coerente na construção do futuro adulto, o que já foi esclarecido por muitos autores(10). Haja vista que residir em assentamentos representa uma vulnerabilidade social e em saúde, possibilitar que o tema, de fato, seja abordado no currículo escolar e no PSE contribuirá significativamente para que os adolescentes e jovens possam ter um desenvolvimento individual e social saudável, minimizando assim os problemas sociais vivenciados, como violência e evasão escolar, Infecções Sexualmente Transmissíveis, tráfico e dependência química. O mais alarmante; $19,05 \%$ dos pesquisados já vivenciaram estudantes fazendo uso de drogas ilícitas no interior da instituição de ensino. Tal achado evidencia a necessidade premente de trabalhar a temática álcool e outras drogas no âmbito escolar de forma transversal em todas as disciplinas.

Regiões de assentamento, entregues ao abandono governamental devido às questões históricas, econômicas e socioculturais, vivenciam déficit de infraestrutura, de educação, saúde e violência, tornando evidente a vulnerabilidade dos estudantes do território em estudo às drogas lícitas e ilícitas ${ }^{(18,4)}$.

$O$ assentamento em estudo não é beneficiado pelo PSE, portanto é importante desenvolver intervenções articulando toda a rede social do adolescente, como família, amigos, professores e pais, buscando o protagonismo juvenil e resguardando as diferenças nas necessidades dos assentados, baseadas na equidade, integralidade e humanização; livres de ideias preconcebidas. O desafio para as ações com esse público é de pensar conjuntamente em estratégias que possam contribuir efetivamente para a prevenção do uso indevido de substâncias lícitas e ilícitas sem interferir no curso natural da vivência e de experiências próprias da adolescência.

Dentre as limitações do presente estudo incluem a natureza da coleta de dados, uma vez que foi baseada no relato verbal, podendo estar sub ou superestimada. Também, há as limitações dos estudos transversais, uma 
vez que não permitem o estabelecimento de relações de causa e efeito.

\section{CONCLUSÃO}

O perfil dos investigados, divergentes do Censo Escolar do Ministério da Educação(21), ratifica a necessidade premente de investimentos em capacitações constantes e valorização profissional que considerem as particularidades do ambiente de trabalho, pois somente assim a escola será considerada um equipamento capaz de prover a prevenção e o desenvolvimento social esperado. Os professores possuem algumas dificuldades formativas, institucionais, culturais e pedagógicas que podem prejudicar a abordagem dessa temática como eixo transversal em sala de aula. Todavia, é premente destacarmos que o trabalho do professor somente será possível e assertivo, se toda a rede social dos adolescentes e adultos jovens estiver engajada com a política institucional e modo de vida do assentamento.

Ainda, é preocupante a porcentagem de professores que vivenciam alunos fazendo uso de drogas nas dependências da escola, o que torna urgente o desenvolvimento de um trabalho coletivo organizado de forma generalizada com uma visão simplista, mas resolutiva sobre o tema.

Atualmente, o piloto do programa Unplugged\#Tamojunto para promoção à saúde e prevenção de drogas em escolas brasileiras tem sido reportando como uma das mais importantes ferramentas na prevenção das drogas, pois potencializa o compromisso dos profissionais da saúde, educação, assistência social, lideranças locais e outros setores para as ações intersetoriais, devendo se unir na perspectiva da organização de uma rede de proteção, participativa e comunitária na busca da superação da problemática das drogas na adolescência e no ambiente escolar.

Por fim, acredita-se que os gestores, impulsionados pelos achados da pesquisa, em parceria com as instituições de ensino e saúde do assentamento, consigam implementar e efetivar o PSE na escola do assentamento, de forma a desenvolver discussões sobre uso e malefícios das drogas de forma articulada entre os professores, estudantes e familiares residentes no assentamento.

\section{Referências}

1. Brasil. Presidência da República. Secretaria Nacional de Políticas sobre Drogas. I Levantamento Nacional sobre o Uso de Álcool, Tabaco e Outras Drogas entre Universitários das 27 Capitais Brasileiras / Secretaria Nacional de Políticas sobre Drogas; GREA/IPQ-HC/FMUSP; organizadores Arthur Guerra de Andrade, Paulina do Carmo Arruda Vieira Duarte, Lúcio Garcia de Oliveira. Brasília. 2010;16.

2. Johnston LD, Miech RA, O'Malley PM, Bachman JG, Schulenberg JE, Patrick ME. Monitoring the Future national survey results on drug use 19752018: Overview, key findings on adolescent drug use. Ann Arbor: Institute for Social Research, University of Michigan.

3. Paul AG. Social stressors and alcohol use among immigrant sexual and gender minority Latinos in a nontraditional settlement state. Substance use \& misuse. 2014; 49(11):1365-75. doi: http://dx.doi.org/10.1590/18069282.64.02.114.

4. Haritha S. The Sex Generation: The impact of teen attitudes, views, and knowledge of contraceptives and sexual activity on the teen pregnancy and STD rates in the United States. Union College. 2011;10(2):12-8. doi: http:// dx.doi.org/10.1590/0104-1169.3059.2363.

5. Marston $M$, et al. Predictors of sexual debut among young adolescents in Nairobi's informal settlements. International perspectives on sexual and reproductive health. 2013;39(1):22. doi: 10.1363 / 3902213.

6. Macedo, JP, et al. Social support, common mental disorder and abusive use of alcohol in rural settlements. Temas em Psicologia, 2018, 26(3): 11231137. $\mathrm{ttp} / / / \mathrm{dx}$.doi.org/10.9788/TP2018.3-01 Pt.

7. Guimarães, RA, et al. Use of illicit drugs by adolescents and young adults of an urban settlement in Brazil. Revista da associação médica brasileira, 2018, 64(2): 114-118. https://doi.org/10.1590/1806-9282.64.02.114

8. Perez ABN, Zerbetto SR, Gonçalves, AMS. Atitudes dos professores de ensino fundamental e médio frente ao uso de substâncias psicoativas. SMAD. Revista eletrônica saúde mental álcool e drogas. 2018;14(1):45-51. doi: 10.11606/issn.1806-6976.smad.2018.000406.

9. Bernardo JF, Mena-chalco JP, Micheli D. Prevenção de drogas em contextos educacionais: uma análise da rede de colaboração entre pesquisadores. Revista Eletrônica de Comunicação, Informação e Inovação em Saúde. 2019;13(3). doi: http://dx.doi.org/10.29397/reciis.v13i3.1561.

10. Lei $n^{\circ} 11.343$, de 23 de Agos. 2006. Institui o Sistema Nacional de Políticas Públicas sobre Drogas - Sisnad. Subchefia de Assuntos Jurídicos. DOU
[Internet]. [acesso em 2019 set 06]. Disponível em: https://goo.gl/22fiJo. 11. Ministério da Saúde. Programa saúde e prevenção na escola. Brasília: MS. 2009. [Acessado em 2014 junho 20]. Disponivel em: http://portal.saude. gov.br/portal/saude/profissional/visualizar_texto.cfm?idtxt=29109\&janela $=1,2009$.

12. Gomes RR. Análise do Censo Escolar da Educação Básica 2016 - Perfil e características dos docentes do estado de Goiás. Instituto mauro borges de estatísticas e estudos socioeconômicos. 2017. [Acesso em: 06 out. 2019]. Disponível em: http://www.imb.go.gov.br/files/docs/releases/censo-escolar-educacao-basica/censo-escolar-educacao-basica-2016.pdf.

13. Costa GLM. Configurações, limites e perspectivas do Ensino Médio no Brasil: qualidade e valorização dos professores. Práxis Educativa. Ponta Grossa. 2013 jun;8(1):85-109. doi: https://doi.org/10.5212/PraxEduc.v.8i1.0004. 14. Costa MA, et al. Formas de violência referidas no cotidiano escolar na visão dos professores de uma escola pública. Rev. Enferm UFSM. 2013 jan;3(1):44-52. doi:10.5902/217976927605.

15. Pereira EF, Teixeira CS, Andrade RD, Bleyer FTS, Lopes AS. Associação entre o perfil de ambiente e condições de trabalho com a percepção de saúde e qualidade de vida em professores de educação básica. Cad. saúde colet. Rio de Janeiro. 2014;22(2). [acesso em 2014 ago 23]. Disponivel em: http://www.scielo.br/scielo.php?script=sci_arttext\&pi$\mathrm{d}=$ S1414-462X2014000200113\&lng=en\&nrm=iso. doi: http://dx.doi.org/10.1590/1414-462X201400020002.

16. Pinto JMR. Remuneração e características do trabalho docente no Brasil: um aporte. Cadernos de Pesquisa. São Paulo. 2011 mai;41(143):606- 639. doi: http://dx.doi.org/10.1590/\$0100-15742011000200014.

17. Assunção $A A$, Abreu MNS. Pressão laboral, saúde e condições de trabalho dos professores da Educação Básica no Brasil. Cadernos de Saúde Pública. 2019;35:517. doi: http://dx.doi.org/10.1590/0102-311x00169517 18. Garcia LSL. A Prevenção ao uso problemático de drogas. 2018.

19. Arraes $\mathrm{CO}$, et al. Masculinidade, vulnerabilidade e prevenção relacionadas às doenças sexualmente transmissiveis/HIV/Aids entre adolescentes do sexo masculino: representações sociais em assentamento da reforma agrária. Rev. Latino-Am. Enfermagem. 2013;21(6)01-08. doi: http://dx.doi. org/10.1590/0104-1169.3059.2363.

20. Secretaria de Atenção à Saúde. Área de Saúde do Adolescente e do Jovem. Marco legal: saúde, um direito de adolescentes. Brasília-DF. 2005;1. 